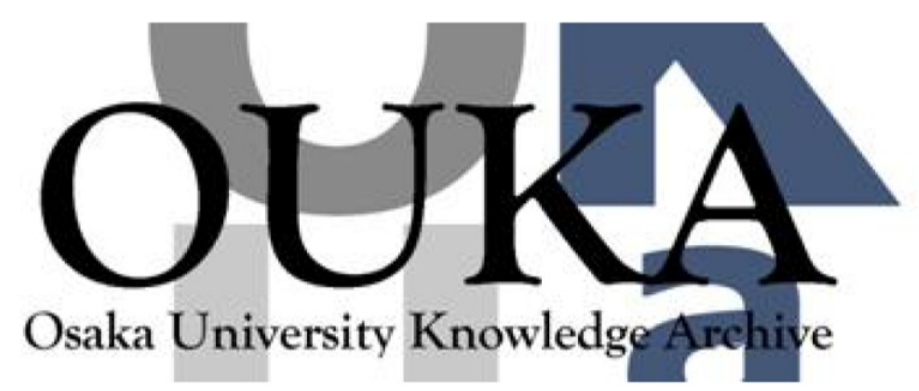

\begin{tabular}{|c|l|}
\hline Title & $\begin{array}{l}\text { Discrete correlators using multiple imaging for } \\
\text { digital optical computing }\end{array}$ \\
\hline Author(s) & Tanida, Jun; Ichioka, Yoshiki \\
\hline Citation & Optics Letters. 16(8) p. 599-p.601 \\
\hline Issue Date & $1991-04-15$ \\
\hline oaire:version VoR \\
\hline URL & https://hdl. handle. net/11094/2959 \\
\hline rights & \\
\hline Note & \\
\hline
\end{tabular}

Osaka University Knowledge Archive : OUKA

https://ir. Library. osaka-u. ac. jp/

Osaka University 


\title{
Discrete correlators using multiple imaging for digital optical computing
}

\author{
Jun Tanida and Yoshiki Ichioka \\ Department of Applied Physics, Faculty of Engineering, Osaka University, 2-1 Yamadaoka, Suita 565, Japan
}

Received December 12, 1990; accepted February 13, 1991

\begin{abstract}
New optical discrete correlators based on multiple-imaging systems with a simple structure and good stability are proposed. The systems are composed of lenses for image duplication and optical shutters for kernel specification. As a result, large throughput can be attained with an incoherent configuration. The throughput of the system is estimated, and several logic operations are demonstrated to verify the usefulness of the proposed systems for digital optical computing. The proposed system will be especially useful for constructing a compact and stable optical computing system.
\end{abstract}

The digital optical computing scheme has advantages of parallelism in optics and flexibility in digital processing. One promising paradigm for optical digital computing is coded pattern processing, such as optical array logic ${ }^{1}$ and symbolic substitution. ${ }^{2}$ In this paradigm, information primitives are encoded into a spatial combination of pixels, set on two-dimensional images, and processed collectively. With the help of information encoding, great flexibility in processing can be obtained with rather simple optical operations.

Discrete correlation is one of the most important operations in the coded pattern processing. This operation is considered as a special case of cross correlation for two two-dimensional images: one image is composed of discrete pixels (called an object), and the other is a set of delta functions offset from each other (called a kernel). The discrete correlation can be achieved by shift and superimpose operations. The capability for dynamic control of the operation is important for discrete correlation because many applications in coded pattern processing require multiple kinds of discrete correlation with different kernels during processing. In addition, the information capacity and light power efficiency during implementation should be considered in practical applications.

There have been many methods proposed to implement discrete correlation. A coherent correlation system $^{3}$ is useful for the operation, and it can be extended to real-time execution by using optical phase conjugation. ${ }^{4}$ Use of diffraction gratings together with the Fourier-filtering technique provides another implementation with a coherent optical system. ${ }^{5}$ In incoherent systems, shadow casting ${ }^{6}$ is an excellent method for moderate resolutional objects, and a reflective optical system ${ }^{7}$ is an alternative. Although they are not proposed for discrete correlation, several methods that use arrays of lenses and prisms for optical perfect shuffle are also useful for the operation..$^{8-10}$

Unfortunately, many of the above methods require complicated optical systems. Complication in the system causes many problems, such as difficulty in fabrication and adjustment, limited information capacity, and poor stability. Although optical shadow casting seems to be a good solution, information capacity is restricted owing to the diffraction effect.

To overcome these problems, the authors propose new methods for discrete correlation with the use of lenses. All information in the system is transferred by imaging, and hence large throughput can be obtained. Since the arrangement of the lenses specifies the operation, various systems for different applications can be constructed with the same fabrication technique. Once the components are built up, the system is stable. No special optical device is required. For these advantages, the proposed methods will be especially useful for constructing a compact and stable optical computing system.

Figure 1 shows a basic optical system for discrete correlation. The system is based on a multiple-imaging system ${ }^{11}$ that consists of two lenses, $L 1$ and L4, and two lens arrays, L2 and L3. The focal lengths of lenses $\mathrm{L} 1, \mathrm{~L} 2, \mathrm{~L} 3$, and L4 are $f_{1}, f_{2}, f_{3}$, and $f_{4}$, respectively. Those lenses are set to form an $8 f$ system.

To achieve the shift function, the spacing between the intermediate imaging plane P2 and lens L3 is adjusted to be slightly different from the focal length of L3. If the spacing is equal to $f_{3}$, an object on P1 is duplicated on P2, and they are again superimposed into a single image on P3. However, if the spacing is different from $f_{3}$, the image on P3 is shifted with the following relations:

$$
\Delta x=\frac{f_{4}}{f_{3}^{2}} X d
$$

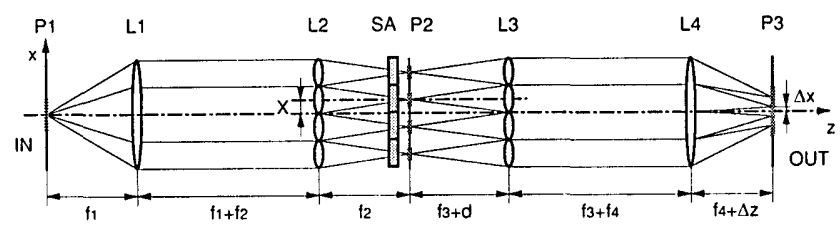

Fig. 1. Basic system of the multiple-imaging discrete correlator. SA is a shutter array for selecting superimposed images. 


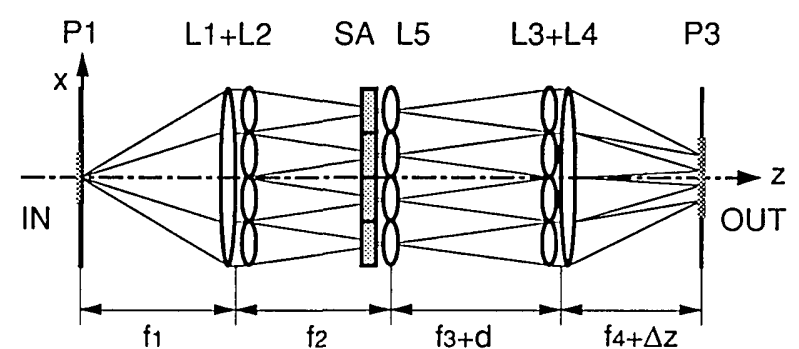

Fig. 2. Power-saving version of the multiple-imaging discrete correlator.

$$
\Delta z=-\frac{f_{4}{ }^{2}}{f_{3}{ }^{2}} d,
$$

where $\Delta x$ and $\Delta z$ are the shift value of the image from the origin of P3 along the $x$ and $z$ directions and $X$ is the distance between the axis of L1 and L 4 and the offset axis of the lenslets in the lens arrays L2 and L3. As seen from Eqs. (1) and (2), the shift along the $x$ axis can be controlled by $d$ by keeping the relation of imaging. Therefore, using lens arrays consisting of small lenslets arranged in a square and selecting the combination of images to be superimposed with an array of optical shutters, we can complete discrete correlation.

Although the basic system is comprehensive, it can be modified to increase the light power efficiency. The light rays passing through L2 diverge, and some of them cannot enter the corresponding lenslet in L3. This causes a decrease of the power efficiency of the system. To avoid this problem, another lens array is inserted in plane P2, which has the function of a field lens. The focal length of lens L5 is

$$
f_{5}=\frac{f_{2}\left(f_{3}+d\right)}{f_{2}+f_{3}+d} .
$$

In addition, lenses L1 and L2 as well as L3 and L4 can be brought into contact. As a result an optical system with high power efficiency is obtained, as shown in Fig. 2.

As seen from Figs. 1 and 2, the optical systems are almost symmetrical. Thus they can be compacted by folding. Figure 3 shows the folded version of the discrete correlator. Unfortunately, the field lens L5 is not at the middle point between the imaging lenses L2 and L3, so that lens L5 cannot be set on the mirror plane in the folded system. This means that there is no place for the field lens or other equivalent reflective elements. As a result, the folded system is inferior to that of Fig. 2 in light power efficiency. However, the system is quite compact. This compactness will be a great advantage in some applications, such as optical circuit modules that compose a large-scale optical computing system. The power loss caused by the beam splitter can be eliminated with a polarization technique.

The resolution limit of the proposed system is determined by that of each lenslet in the lens array. For simplicity, consider the system in Fig. 2 with $f_{1}=f_{4}$ and $f_{2}=f_{3}$. Then the minimum resolution length on $\mathrm{P} 1, x_{\min }$, is

$$
x_{\min }=\frac{1.22 f_{1} \lambda}{D_{2}},
$$

where $\lambda$ is the wavelength of light and $D_{2}$ is the aperture size of a lenslet in L2 and L3. The object size to be correlated is determined by the aperture size of the lenslet in L5, $D_{5}$. The maximum object size $S_{\max }$ is

$$
S_{\max }=\frac{f_{1} D_{5}}{f_{2}} \text {. }
$$

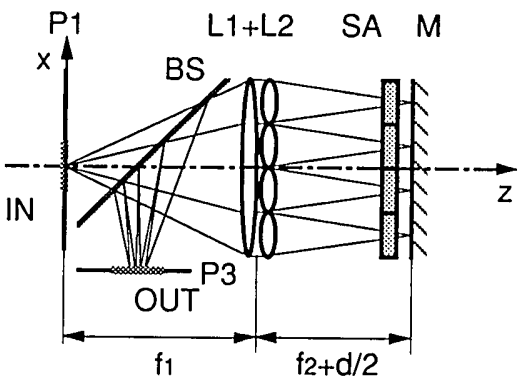

Fig. 3. Folded version of the multiple-imaging discrete correlator. BS, beam splitter; $\mathrm{M}$, mirror.

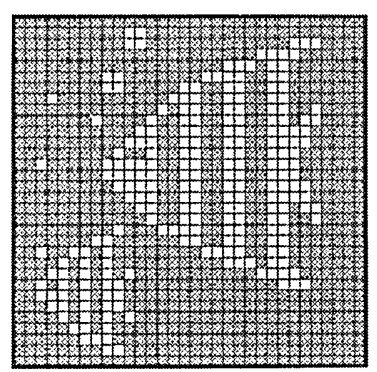

(a)

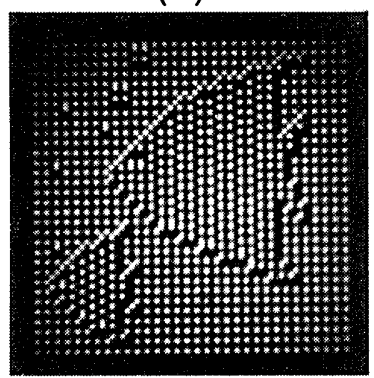

(c)

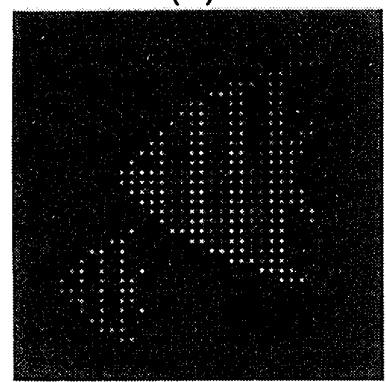

(e)

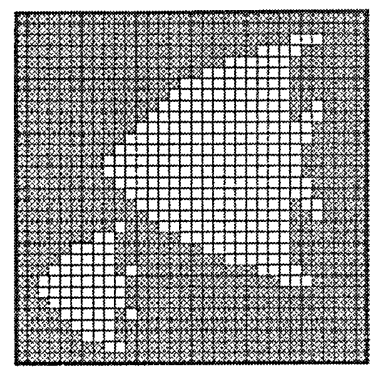

(b)

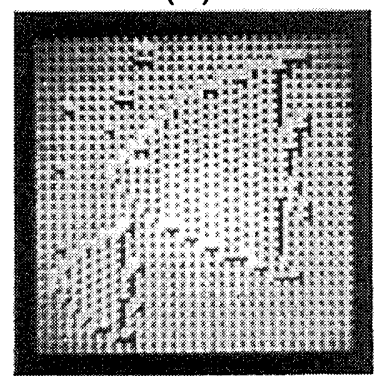

(d)

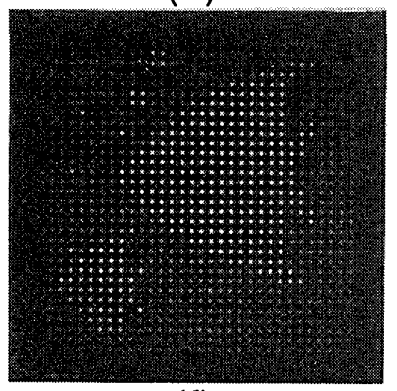

(f)
Fig. 4. Experimental results of logical operations: (a) and (b) are input images for operations, (c) and (d) are correlated results of the coded object with kernels for AND and OR operations, and (e) and (f) are the final results of AND and oR operations after sampling. 
Consequently, the maximum number of data points processed in this optical system for one dimension, $N_{\max }$, is

$$
N_{\max }=\frac{S_{\max }}{x_{\min }}=\frac{D_{2} D_{5}}{1.22 f_{2} \lambda} .
$$

For $D_{2}=D_{5}=2 \mathrm{~mm}, \lambda=500 \mathrm{~nm}$, and $f_{2}=1.6 \mathrm{~mm}$, $N_{\max }$ is equal to 4098 , so that approximately $13,000,000$ data points can be processed on a circular object.

Here, the effect of scale should be commented on. If all the components except the wavelength are reduced by a certain factor, $N_{\max }$ is decreased by the factor. This means that reduction of the system size results in a decrease of the information capacity of the system. Therefore it is important to determine system size based on the purpose of the system.

To verify the performance of the proposed method, several logic operations are executed based on optical array logic. A folded discrete correlator is used for the experiments. Figure 4 shows the results of AND and OR operations for two binary images. The binary images consisting of $32 \times 32$ pixels in Figs. 4(a) and 4(b) are encoded into an object. Individual pixel pairs at the sample positions in the input images are converted into spatial patterns. In the spatial pattern, a quarter-pixel size of the window is positioned on any one of the corners of the individual pixel according to the combination of the pixel values.

The coded object is correlated by the folded discrete correlator as shown in Figs. 4(c) and 4(d). The object size and the cell size are $4.0 \mathrm{~mm}$ and $65.2 \mu \mathrm{m}$, respectively. The specifications of the lenses used are $f_{1}=$ $300 \mathrm{~mm}, D_{1}=50 \mathrm{~mm}, f_{2}=250 \mathrm{~mm}$, and $D_{2}=10 \mathrm{~mm}$. The lens L2 is composed of $2 \times 2$ lenslets with an offset of $11 \mathrm{~mm}$. The kernels are a single delta function and a set of triple delta functions arranged in the manner of a reversed letter L, which are used for AND and OR operations, respectively. These kernels are specified by the opening pattern of the optical shutters.
In optical array logic, results of parallel operations are obtained by spatial sampling of the correlated image. Figures $4(\mathrm{e})$ and $4(\mathrm{f})$ are the final results of parallel AND and OR operations after sampling. By using different opening patterns of the optical shutters, we can execute various kinds of parallel logical operation with the optical system.

In conclusion, new incoherent optical systems for discrete correlation have been proposed, and their performance has been estimated. The principle of the method has been verified by logical operations. This system is useful for various kinds of fundamental, parallel optical computing such as optical array logic, symbolic substitution, and free-space optical interconnection. Since the information capacity to be dealt with decreases as the optical system is reduced in scale, the scale of the system should be determined dependent on its applications.

\section{References}

1. J. Tanida and Y. Ichioka, J. Opt. Soc. Am. A 2, 1245 (1985).

2. K.-H. Brenner, A. Huang, and N. Streibl, Appl. Opt. 25, 3054 (1986).

3. J. W. Goodman, Introduction to Fourier Optics (McGraw-Hill, New York, 1968), Chap. 7.

4. G. Eichmann, Y. Li, and R. R. Alfano, Appl. Opt. 26, 194 (1987).

5. R. Thalmann, G. Pedrini, B. Acklin, and R. Dändliker, Proc. Soc. Photo-Opt. Instrum. Eng. 963, 635 (1988).

6. J. Tanida and Y. Ichioka, J. Opt. Soc. Am. 73, 800 (1983).

7. J. Tanida, J. Nakagawa, and Y. Ichioka, Appl. Opt. 27, 3819 (1988).

8. A. Lohmann, Appl. Opt. 25, 1543 (1985).

9. C. W. Stirk, R. A. Athale, and M. W. Haney, Appl. Opt. 27, 202 (1988).

10. M. W. Haney and J. J. Levy, in Conference Record of 1990 International Topical Meeting on Optical Computing (Japan Society of Applied Physics, Kobe, Japan, 1990), p. 85.

11. K. Hamanaka, H. Nemoto, M. Oikawa, E. Okuda, and T. Kishimoto, Appl. Opt. 29, 4064 (1990). 